\title{
Core bone diameter in an organic implant-less technique affecting the biomechanical properties of the anterior cruciate ligament fixation; an in-vitro study
}

\author{
Running Title: \\ Geometry effects on BASHTI technique
}

\author{
Authors / Affiliations: \\ Mahdi Mohseni, M.Sc. student \\ Research Assistant, Department of Mechanical Engineering, Sharif University of Technology, \\ Tehran, IR, mahdi.mohseni@mech.sharif.edu, Phone: +989135335667 \\ Amir Nourani*, Ph.D.
}

Assistant Professor, Department of Mechanical Engineering, Sharif University of Technology, Tehran, IR, nourani@sharif.edu, Phone: +982166165687

Hossein Korani, M.Sc. student

Research Assistant, School of Mechanical Engineering, College of Engineering, University of Tehran, Tehran, IR, Korani@ut.ac.ir, Phone: +989129494789

Hadi Moeinnia, Ph.D. student

Research Assistant, Mechatronics System Engineering, Simon Fraser University, Burnaby, BC V5A 1S6, Canada, h.moein@ student.sharif.edu, Phone: +989375151931

Amirhossein Borjali, M.Sc.

Research Assistant, Department of Mechanical Engineering, Sharif University of Technology, Tehran, IR, a.borjali15@ student.sharif.ir, Phone: +989393962780

Narges Ghias, B.Sc. student

Research Assistant, Department of Mechanical Engineering, Sharif University of Technology, Tehran, IR, narges.ghias1395@sharif.ir, Phone: +989133178264

Mahmoud Chizari, Ph.D.

Senior Lecturer, School of engineering and computer sciences, University of Hertfordshire, College Ln, Hatfield AL10 9AB, UK, m.chizari@ herts.ac.uk, Phone: +44(0)1707284000

*Corresponding author

Amir Nourani

E-mail: nourani@sharif.edu

Phone: +982166165687, Fax: +982166000021

\section{Acknowledgements}

The authors sincerely appreciate all the help and guidance of Dr. Farzam Farahmand, director of the Biomechanics Laboratory of Sharif University of Technology. 


\section{Author contributions}

47 All authors contributed to the study conception and design. Material preparation, data collection

48 and analysis were performed by Mahdi Mohseni, Hossein Korani, Hadi Moeinnia, Amirhossein

49 Borjali and Narges Ghias. The first draft of the manuscript was written by Mahdi Mohseni, revised

50 by Amir Nourani and Mahmoud Chizari and all authors commented on previous versions of the

51 manuscript. All authors read and approved the final manuscript.

52

\section{Declarations}

54 The authors declare that they have no conflict of interest.

55

\section{Compliance with Ethical Standards}

57 Declarations of interest: None

58 This research did not receive any specific grant from funding agencies in the public, commercial, 59 or not-for-profit sectors.

60 
bioRxiv preprint doi: https://doi.org/10.1101/2021.07.12.452098; this version posted July 13, 2021. The copyright holder for this preprint (which

was not certified by peer review) is the author/funder, who has granted bioRxiv a license to display the preprint in perpetuity. It is made available under aCC-BY 4.0 International license.

\section{Highlights}

62 - A new implant-less technique was used to reconstruct anterior cruciate ligament.

63 - Artificial bone and fresh animal soft tissue used to simulate the fixation process.

64 - Loading condition were carefully chosen to simulate the post-operation.

65

- Components geometry had direct effect on biomechanical properties of the fixation.

66

- Optimum geometry was found trough an experimental examination. 


\section{Abstract}

Background: Bone and site hold tendon inside (BASHTI) is an implant-less technique that can

69 solve some of the problems associated with other anterior cruciate ligament (ACL) reconstructive methods. This study aims to investigate the effect of core bone diameter variation on the

71 biomechanical properties of a reconstructed ACL using BASHTI technique.

72 Methods: A number of 15 laboratory samples of reconstructed ACL were built using bovine

73 digital tendons and Sawbones blocks. Samples were divided into three groups with different core

74 bone diameters of $8 \mathrm{~mm}, 8.5 \mathrm{~mm}$, and $9 \mathrm{~mm}$. The double-stranded tendon size and bone tunnel

75 diameter were $8 \mathrm{~mm}$ and $10 \mathrm{~mm}$, respectively. A loading scenario consisting of two cyclic loadings

76 followed by a single cycle pull-out loading was applied to the samples simulating the after-surgery

77 loading conditions to observe the fixation strength.

78 Results: Results showed that the core bone diameter had a significant effect on the failure mode

79 of the samples $(P=0.006)$ and their fixation strength $(P<0.001)$. Also, it was observed that the

80 engaging length and the average cyclic stiffness (ACS) of them were influenced by the core bone

81 diameter significantly (engaging length: $P=0.001$, ACS: $P=0.007$ ), but its effect on the average

82 pull-out stiffness was not significant $(P=0.053)$.

83 Conclusions: It was concluded that core bone diameter variation has a significant impact on the

84 mechanical properties of ACL reconstruction when BASHTI technique is used, and it should be 85 noted for surgeons who use BASHTI technique. 

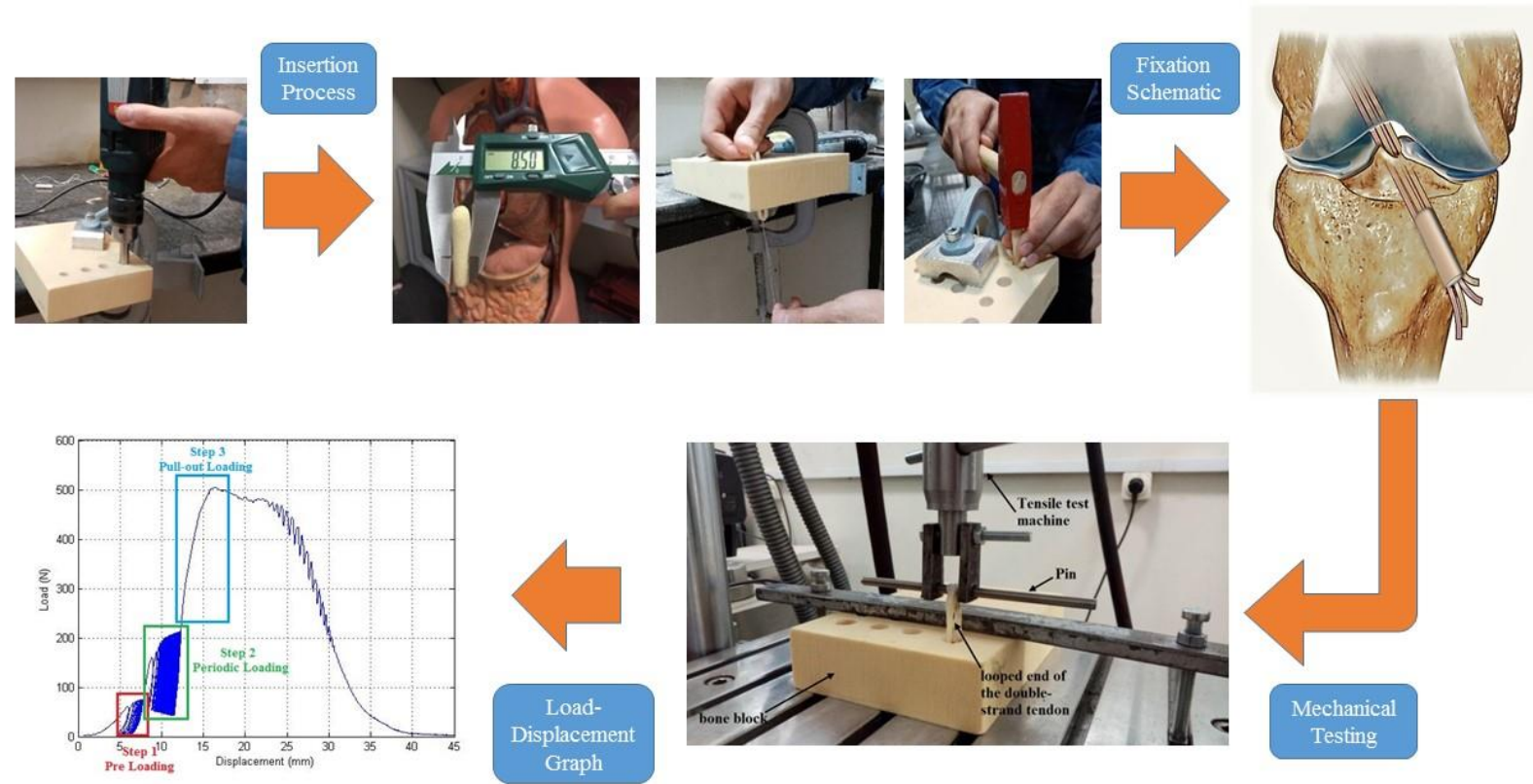

86

87 Keywords: BASHTI technique, Core bone diameter, ACL reconstruction, Geometric parameters,

Fixation strength 


\section{Introduction}

90

91

92

Ligaments are fibrous bands connecting two bones, capable of undergoing tension and a ligament rupture is a common injury in the human body. This injury can be a result of extreme conditions caused by high pressures or impact, usually during sports activities ${ }^{1}$. Various techniques have been proposed to reconstruct a ruptured ligament, and most of them include an external implant. Every technique has been proved to have its own advantages and disadvantages. There are some different methods to reconstruct a ruptured ACL, such as using some sutures to hold the ligament next to the bone (suture anchor) ${ }^{2}$ or fixing the ligament via a button (cortical button) ${ }^{3}$. However, the most common technique is using an interference screw ${ }^{4}$. This is a reliable technique with proper strength, in which a screw is used to fix the tissue in a bone tunnel ${ }^{4-7}$.

The mentioned conventional techniques for ACL reconstruction still comes with some problems. One of them is the high cost of using an external implant like the interference screw. Also, using these external implants may cause some side effects such as soft tissue rotation ${ }^{8}$, bone tunnel widening ${ }^{9}$ and interfering in magnetic resonance imaging (MRI) ${ }^{10}$. To solve these problems, recent studies have been focused on new implant-free techniques for ACL reconstruction, such as the press-fit technique that uses the cylindrical bone block of patellar attached to the tendon graft to fix the connective tissue ${ }^{11}$. However, using this method may cause some problems such as pain in the patellar donor area ${ }^{12}$.

A new implant-free approach presented in this area is bone and site hold tendon inside (BASHTI). In this technique, neither an external implant nor the patellar bone but the patient's tibia bone is used to perform the reconstruction ${ }^{13}$. Therefore, no sign of allergic reaction is observed, and the costs of using an external implant made of expensive metals or biodegradable polymers are eliminated ${ }^{11}$. Also, there would not be an implant to intervene with MRI images. To 
112 carry out the process, a specially designed drill bit is utilized on the bone to provide a tunnel and 113 a cylindrical bone block called the core bone. The core bone is later used to fix the ligament inside

114 the tunnel instead of using an external implant like an interference screw. The healing process in

115 BASHTI technique can be faster ${ }^{11}$. Hence, efforts have been made to improve this fixation 116 technique.

BASHTI technique was introduced in a research made on bovine bones and digital tendons harvested from bovine feet. The research experimentally compared the BASHTI results with the 119 interference screw fixation. The study concluded that the strength of BASHTI technique is as high 120 as an interference screw ${ }^{13}$. Due to previous studies, tendon compression is defined as a 121 dimensionless parameter related to the amount of volume strain of the tendon in this fixation 122 technique. Recently, it was observed that this parameter significantly affects the strength of BASHTI fixation with an experimental study using bovine digital tendons and artificial bones. It

124 was showed that increasing the tendon compression up to an optimum value could improve the 125 fixation strength ${ }^{14}$.

Furthermore, an investigation was performed on the effect of using a sheathed core bone on

127 the biomechanical properties of the ligament fixation created by the BASHTI technique, and it was

128 observed that using these sheathes could help to increase the length of conflict between the core

129 bone, the ligament and the tunnel, resulting in a stronger fixation and also decrease the tunnel 130 widening and core bone fracture during the insertion procedure ${ }^{15}$. Lastly, Nourani et al. studied 131 two insertion procedures to build the BASHTI fixation for biceps tendon reconstruction using 132 different frequencies. Results showed that using frequencies below 300 beats per minute would 133 improve the strength of the BASHTI fixation significantly ${ }^{16}$. All of the previously mentioned 
134

135

studies used Sawbones artificial bone blocks and bovine digital tendon to simulate the real conditions.

The purpose of this study was to evaluate the effect of core bone diameter on the mechanical properties of BASHTI technique under loading conditions similar to real ACL loadings. Geometrical conditions, including the diameters of the core bone and the tendon used to perform the reconstruction, are to be studied. These parameters can affect the amount of compression the tendon withstands. This is an experimental study about the effect of core bone diameter on the strength and stiffness of BASHTI fixation for ACL reconstruction, and also on the engaging length between the core bone and the fixed ligament in the tunnel that could affect the strength of fixation and speed up the healing process.

\section{Materials and Methods}

\subsection{Materials and Specimen Preparation}

Bovine digital tendons were harvested using surgical instruments and stored at $-20^{\circ} \mathrm{C}$ to ensure its biomechanical properties remain unchanged ${ }^{17-19}$ (Fig. 1 a). They were used to model human ligament ${ }^{14,19}$. After the harvesting process, the tendon was trimmed to an identical size using surgical blade (Fig. 1 b). As a choice in ACL surgery, it was decided to use tendons with a doublestranded diameter of $8 \mathrm{~mm}$. The tendons were kept moist by spraying water during preparation and testing procedures maintain their mechanical properties ${ }^{18}$. Moreover, Sawbones Polyurethane blocks (1522-03, Sawbones Europe AB, Malmo, Sweden) with a density of $320 \mathrm{Kg} / \mathrm{m}^{3}\left(20 \mathrm{lb} / \mathrm{ft}^{3}\right)$ were used as the artificial bone to represent young human tibia bone ${ }^{14,19}$ (Fig. 1 c). 
The artificial bone was tunneled using a specially designed cannulated drill bit with an

156 outer diameter of $10 \mathrm{~mm}$. The inside core bone of the tunnel was extracted after tunneling (Fig. 1

$157 \mathrm{~d}$ and e). To perform the reconstruction, a double-stranded tendon was entered the tunnel by using

158 a suture, and the extracted core bone was hammered into it with a frequency of lower than 300

159 beats per minute ${ }^{16}$ (Fig. 2 a and b). The first few millimeters of the core bone were chamfered to

160 more easily enter the tunnel. The core bone could not fully penetrate the tunnel, and its end part

161 was broken after hammer impacts and it could affect the strength of fixation. So the length of the

162 core bone that successfully entered the tunnel was measured after every experiment and reported

163 as the engaging length to examine its effect on the fixation strength.

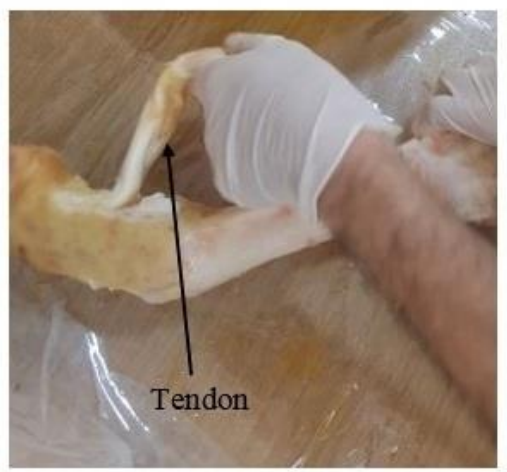

a

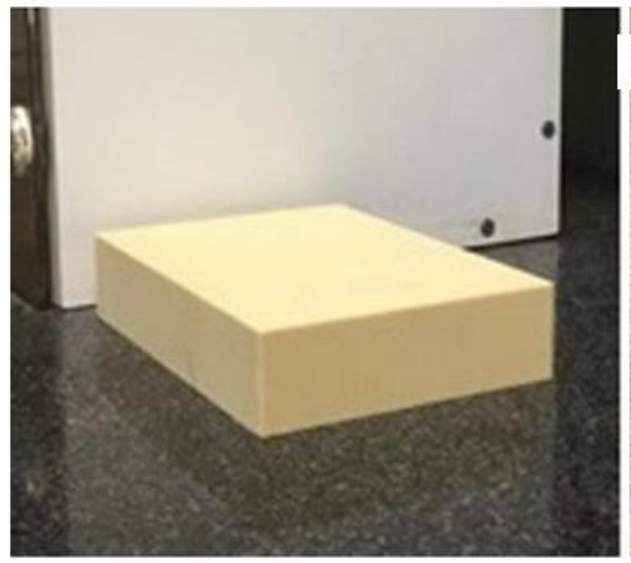

$\mathrm{C}$

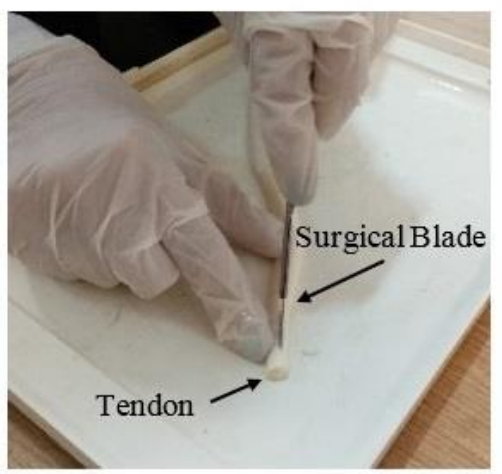

b

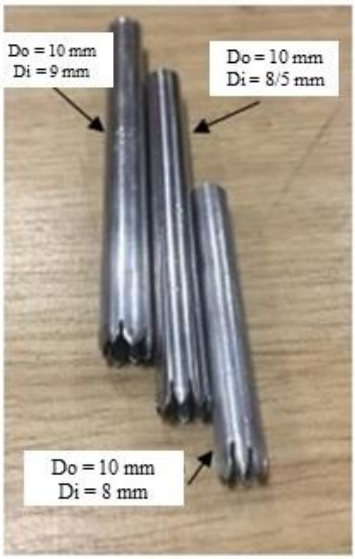

d

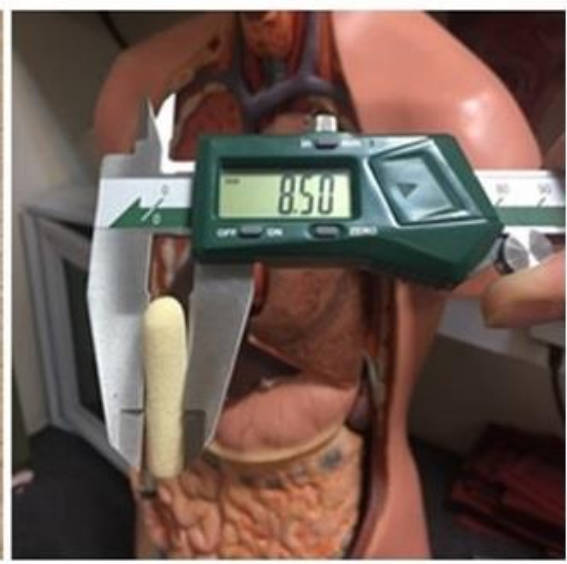

e 
Fig. 1 a. Bovine digital tendon extraction, b. Adjusting the tendon diameter using a surgical blade, drill bits with an outer diameter of $10 \mathrm{~mm}$ and inner diameters of $9 \mathrm{~mm}, 8.5 \mathrm{~mm}$, and $8 \mathrm{~mm}$ were used to extract the core bones, e. The core bone extracted from the artificial bone block with the desired diameter.

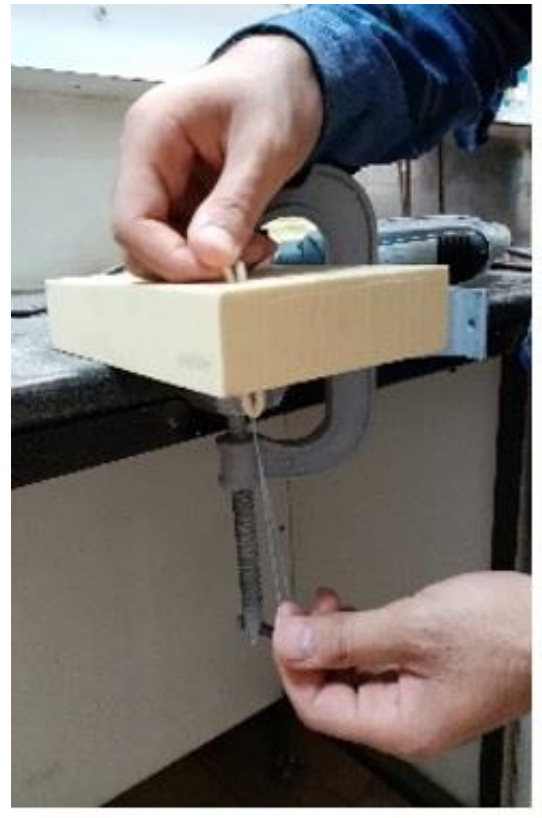

a

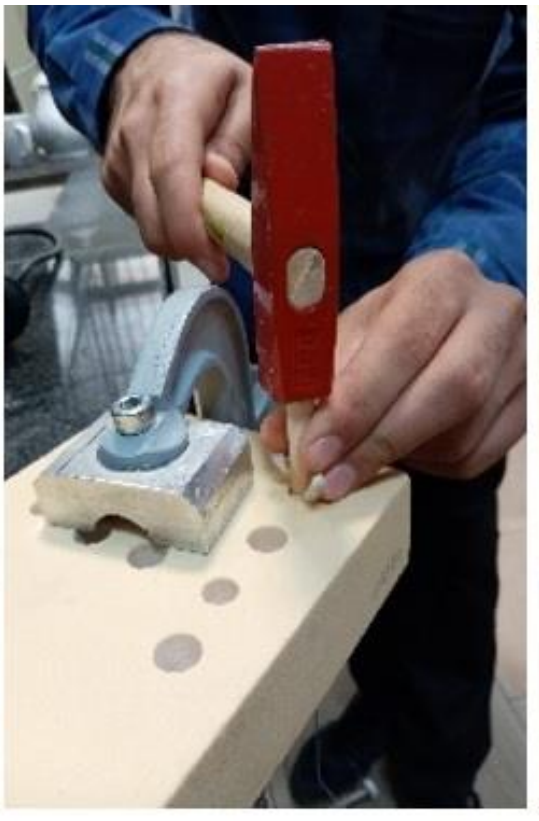

b

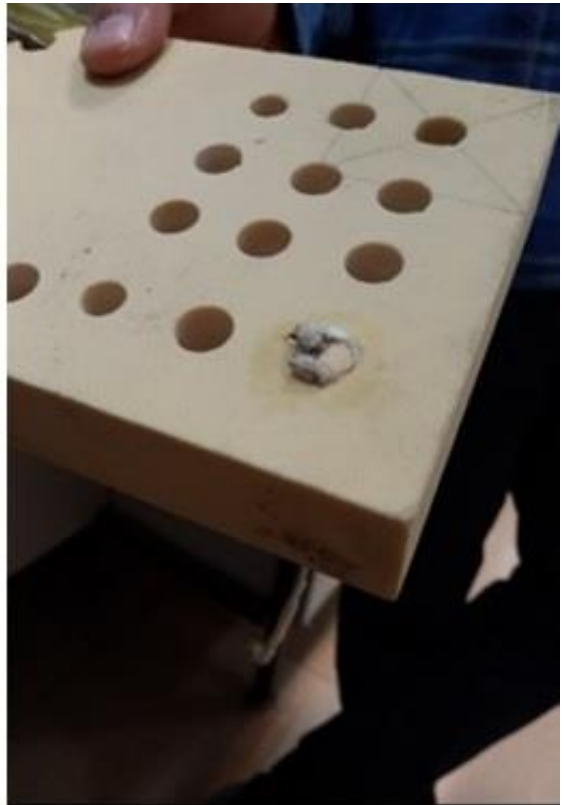

c 
180 Fig. 2 a. the double-strand tendon has entered the tunnel with a suture, b. The core bone was

181 hammered into the tunnel, c. The tendon and the core bone were entered into the tunnel, and the

182 laboratory sample of BASHTI fixation was prepared.

\subsection{Biomechanical Testing}

185 The looped end of the double-stranded tendon was attached to the Zwick-Roell Amsler HCT 25-

186400 tensile testing machine using a pin while the sawbones block was fixed on the table of the

187 testing machine (Fig. 3). A pull-out scenario was used so that the fixation underwent three levels

188 of loading, simulating the real loading conditions of ACL. For the preload, the fixation was

189 subjected to 10 cycles ranging from 10 to $50 \mathrm{~N}$ with a frequency of $0.1 \mathrm{~Hz}$. Afterward, a periodic

190 loading with 150 cycles between 50 to $200 \mathrm{~N}$ at a frequency of $0.5 \mathrm{~Hz}$ was exerted on the fixation

$191{ }^{20}$. In case that the construction sustained these two levels, the machine immediately pulled the

192 tendon with a rate of $20 \mathrm{~mm} / \mathrm{min}$, until the fixation failed ${ }^{19}$. Each experiment was repeated five

193 times to validate the repeatability of the experiment. 


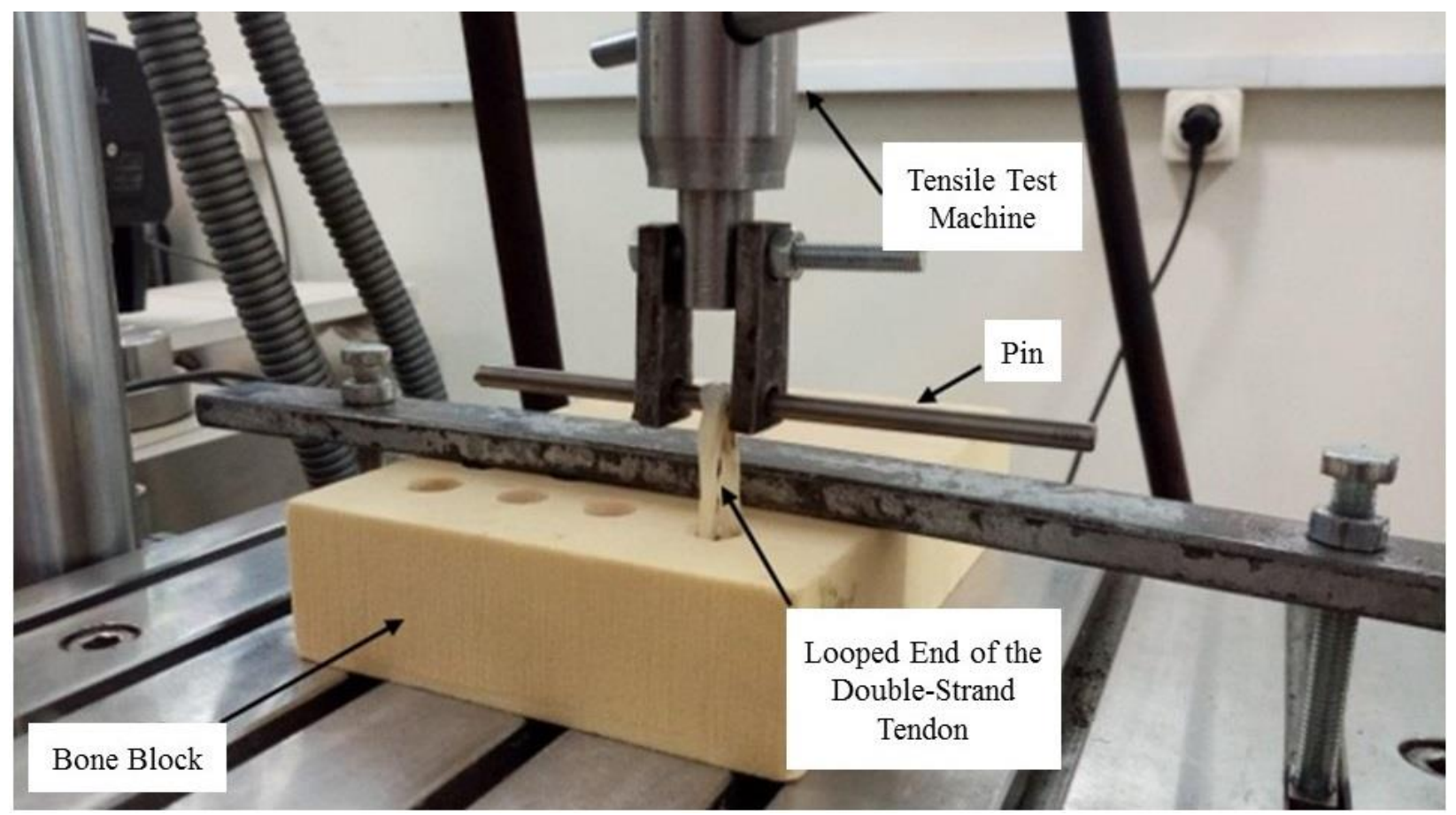

Fig. 3 Testing a typical sample using BASHTI technique. It was assumed that when the tendon displacement became more than $10 \mathrm{~mm}$, the failure mode occurs on the fixation site.

\subsection{Statistical Methods}

The 95\% confidence intervals of the results were calculated using Student's t distribution. Also, ANOVA one-way method was used to analyze the biomechanical properties results. The failure mode results were analyzed using Chi-Square statistic method, which is commonly used for testing the relationships between categorical variables. In this regard, probability value (P-value) was supposed to compare different groups. In case if the P-value is equal or less than 0.05 , it means the differences between the two groups with $95 \%$ confidence are significant.

\section{Results}


208 The failure mode results of all groups are shown in Fig. 4 a. It was observed that about $47 \%$ of the

209 samples failed during the cyclic loading and did not reach the pull-out loading step. According to

210 these results, all of the Group 3 samples failed in the cyclic loading step (second loading step),

211 while the loading step that caused all of Group 2 samples to fail was pull-out loading (third and

212 last step). Only among the specimens in Group 1, both of the above conditions were observed,

213 with two specimens failing in cyclic loading and three specimens in the tensile loading step. Duo

214 to these results, statistical analysis showed that the core bone diameter affects the failure of the

215 samples significantly $(\mathrm{P}=0.006)$. Also, all of the failure modes occurred at the fixation site (i.e.,

216 the tendon and core bone slipped out of the bone tunnel without tendon rupture) (Fig. 3), and no

217 tendon rupture was observed. One typical load-displacement graph (i.e., the fifth test of Group 2)

218 is shown in Fig. 4 b. The three loading steps are illustrated in this figure, and it can be seen how

219 much displacement has been made in the sample at each loading step. 

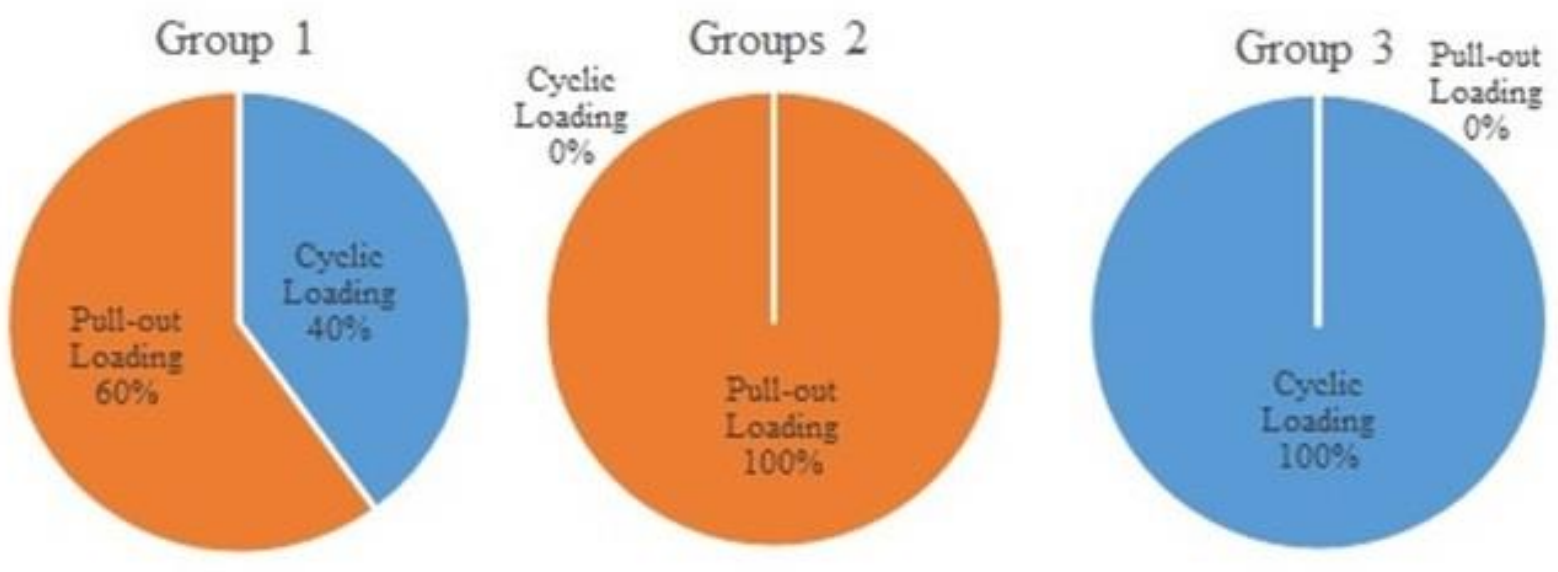

a

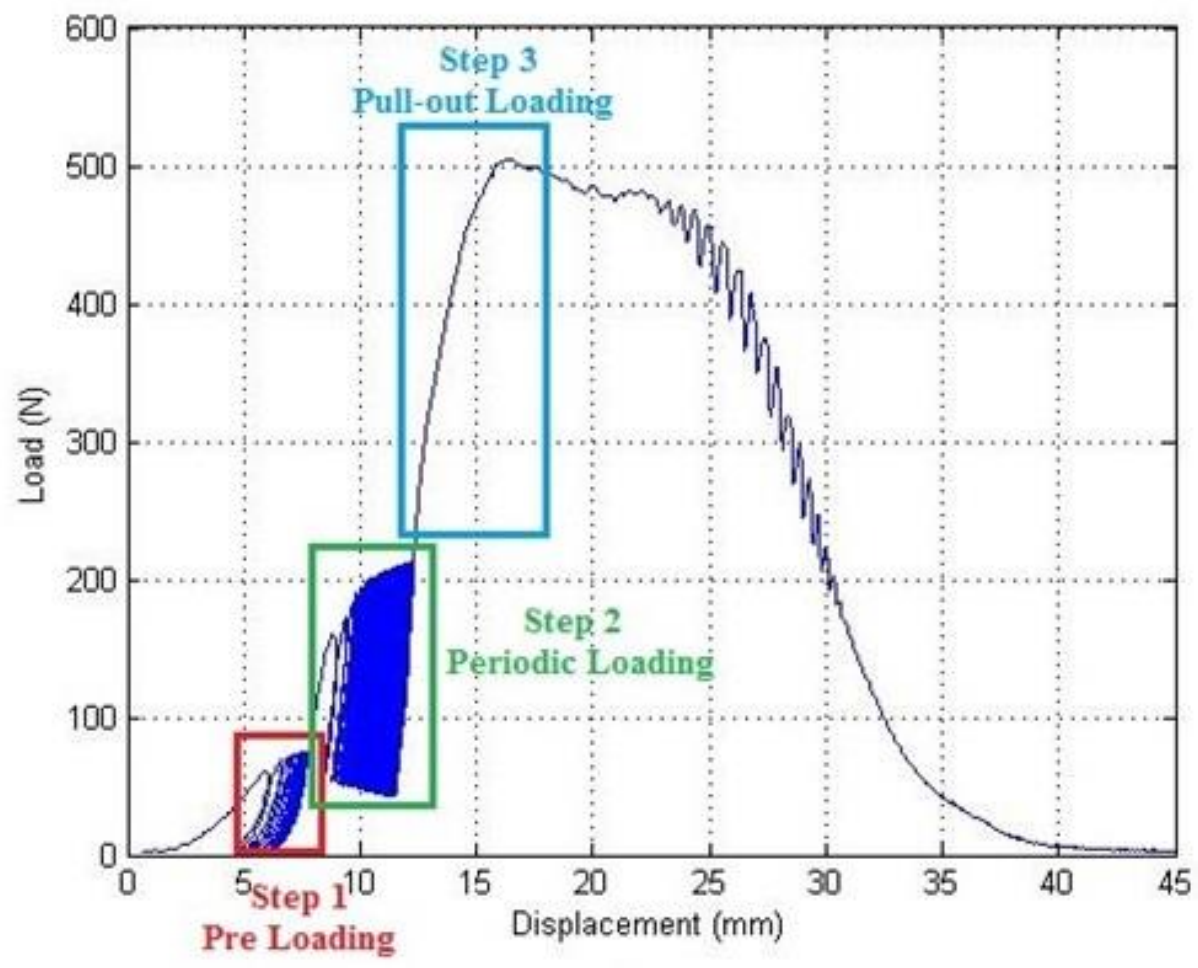

$\mathrm{b}$

222 Fig. 4 a. Loading step that samples reached the failure in Groups 1, 2 and 3, b. Three loading steps

223 load-displacement result of the fifth sample in Group 2. 
The biomechanical results are available in Table 1. It was assumed that when the

226 deformation from the beginning of the second cyclic loading step reached $10 \mathrm{~mm}$ (that is about

$22730 \%$ of the average initial ACL length ${ }^{21}$ ), the fixation is failed (i.e., the failure mode is fixation

228 failure). In this case, the maximum load in the $10 \mathrm{~mm}$ range has been reported as the fixation

229 maximum strength. Also, the average cyclic stiffness (ACS) was defined using the following

230 equation:

$231 \quad A C S=\frac{F_{C}}{D_{C} /_{N_{C}}}(N / \mathrm{mm})$

232 where $F_{C}$ is the amplitude of the periodic loading (e.g., it is equal to $150 \mathrm{~N}$ for a cyclic loading

233 between $200 \mathrm{~N}$ and $50 \mathrm{~N}$ ), $D_{C}$ is the final pure displacement of the looped end of the tendon in the

234 periodic loading step, and $N_{C}$ is the number of completed cycles. The ACS value quantifies the

235 behavior of the reconstructed ACL against active extension loading during the post-surgical and

236 rehabilitation period.

Also, the average slope of the load-displacement curve in the linear region of the pull-out

238 loading step was reported as the average pull-out stiffness (APS) that implies the reconstructed

239 ACL resistance to sudden impact loading. Note that ACS and APS values were calculated only for

240 the specimens that fulfilled the cyclic loading step. Subsequently, since none of the Group 3

241 samples reached the pull-out loading step, no values were reported as the stiffness for this group.

242 Finally, the length of the core bone that successfully entered the tunnel without any fracture and

243 slipped out of it after the failure of the structure was measured and reported as the engaging length

244 in Table 1. 
Table 1. The biomechanical properties of each group calculated from the Load-Displacement curve of each sample with $95 \%$ Confidence Interval

\begin{tabular}{|c|c|c|c|c|}
\hline Group & Maximum & ACS (N/mm) & Average Pull-Out & Engaging Length \\
& Strength (N) & & Stiffness (N/mm) & $(\mathbf{m m})$ \\
\hline 1 & $193 \pm 33$ & $2118 \pm 522$ & $114.4 \pm 40.8$ & $11.4 \pm 5.2$ \\
\hline 2 & $360 \pm 123$ & $3270 \pm 574$ & $79 \pm 27$ & $9.6 \pm 0.5$ \\
\hline 3 & $137 \pm 62$ & $-^{*}$ & $-^{*}$ & $23.2 \pm 9.5$ \\
\hline
\end{tabular}

* No value was reported since none of the Group 3 samples reached the pull-out loading step. not affect the APS of the samples.

Table 2. The results of statistical analysis for the effect on core bone diameter on the biomechanical

257 properties of BASHTI technique. A P-value less than 0.05 considered statistically significant

\begin{tabular}{|c|c|c|c|c|}
\hline Biomechanical & Maximum & ACS (N/mm) & APS (N/mm) & Engaging \\
Property & Strength (N) & & Length (mm) \\
\hline P-value & 0.000 & 0.007 & 0.053 & 0.002 \\
\hline
\end{tabular}




\section{Discussion}

According to the results obtained, it could be concluded that the core bone diameter had a significant effect on the failure mode of the samples $(P=0.006)$. In Group 3 samples (i.e., $8 \mathrm{~mm}$ core bone), since the core bone failed to compress the $8 \mathrm{~mm}$ tendon into the tunnel wall properly, all of the specimens failed in the main cyclic not even reached to step 3 pull-out loading. While in

264 Group 2 samples (i.e., $8.5 \mathrm{~mm}$ core bone), the core bone managed to compress the tendon fibers into the tunnel wall, and all of the samples failed in the third loading step. Moreover, in Group 1 samples (i.e., $9 \mathrm{~mm}$ core bone), only 60 percent of specimens reached the step 3 loading (Fig. 4 a), and 40 percent failed to do so. The reason for this occurrence is over-compression. In other words,

268 since the diameter of the tunnel was fixed, by increasing the core bone diameter, the compression between core bone, tendon, and tunnel wall increased significantly. As a consequence, the overcompression damaged the tendon fibers and made its mechanical properties weaker. So, some

271 specimens failed in the cyclic loading step. It is in agreement with the results of previous studies 272 on this method ${ }^{14}$.

Also, it was observed that the core bone diameter had a significant effect on the maximum

274 strength of the reconstructed ACL $(\mathrm{P}$-value $=0.000)$. Based on previous studies, the strength of a 275 reconstructed ACL should be more than $200 \mathrm{~N}$, which is the physiologic requirement for the leg's 276 passive motion during rehabilitation ${ }^{22}$. It was observed that just the results of Group 2 samples

277 had the strength values higher than the $200 \mathrm{~N}$ limit. The critical point was that as the core bone 278 diameter increased from $8 \mathrm{~mm}$ to $8.5 \mathrm{~mm}$, the strength of the structure increased. When the 279 diameter increased to $9 \mathrm{~mm}$, due to over-compression, the result showed a lower strength than 8.5 
281 the tendon size from $8.5 \mathrm{~mm}$ to $9 \mathrm{~mm}$ would decrease the fixation strength, and the core bone with $8.5 \mathrm{~mm}$ is the best choice for $8 \mathrm{~mm}$ tendons. reconstructed ACL $(\mathrm{P}$-value $=0.007)$. The ACS means the resistance of the tendon graft to the displacement in cyclic loading, and it shows the functionality of the reconstruction in daily activities. It was shown that the BASHTI structure for $8 \mathrm{~mm}$ tendon had the best ACS when the core bone diameter was $8.5 \mathrm{~mm}$. As a result, this core bone diameter provided better properties against the active extension loadings immediately after the ACL reconstruction surgery and during the rehabilitation period. On the other hand, the core bone diameter had an insignificant effect on the APS (P-value $=0.053)$. It is noteworthy that the APS is related to the resistance of the reconstructed connective tissue to sudden impact loadings (e.g., heavy activities in soccer, 292 basketball). samples $(P=0.002)$. Still, there was no significant difference between the engaging length of 295 Groups 1 and $2(P=0.431)$. It is believed that when the core bone diameter increases and the tunnel size kept constant, the tendon compression and the friction between tendon and tunnel wall 297 would increase ${ }^{14}$. Thus, a higher amount of hammer impacts is needed to insert the core bone 298 inside the tunnel. This may increase the risk of core bone fracture, and therefore a shorter length 299 of core bone would be entered into the tunnel. According to Table 1, since the Group 3 had the 300 smallest core bone diameter, no excessive hammer impacts were needed to insert the core bone 301 into the tunnel; hence, the engaging length in this group was significantly higher from Groups 1 and 2. Besides, in this group, although the core bone did not encounter massive strikes during the insertion process, and almost remained undamaged after the fixation, since there were not 
sufficient forces to fix the tendon into the tunnel, all of the samples in this group failed in the main cyclic loading step.

It is concluded that the geometrical parameters had a significant effect on the

307 biomechanical properties of reconstructed ACL with BASHTI technique. While increasing the 308 core bone diameter could improve the strength and stiffness of the ACL, this diameter exceeding 309 its critical value would reduce the biomechanical properties of the reconstructed ACL due to over310 compression. This study had some limitations that might affect the results; such as lack of human

311 tibia bone and soft tissue for more accurate modeling, absence of a comparison of the results with 312 interference screw as a gold standard of ACL reconstruction in the same conditions, and absence 313 of investigation of the interactions between core bone and tendon diameters.

\section{Conclusions}

316 This study aimed to find out the effect of core bone diameter on the biomechanical properties of

317 BASHTI fixation, which is an implant-less technique for ACL reconstruction. It also investigated

318 the optimum size of the core bone for a specific ligament size which was proven to have the best

319 outcome in previous studies. A series of experimental examinations were performed using 320 BASHTI technique to model the reconstructed ACL fixation and loading condition. It was 321 observed that the core bone diameter had a significant effect on almost all of the biomechanical 322 properties of the reconstructed samples. The study introduced a threshold and a critical value for 323 the optimum diameter of the core bone. Although this is not a clinical study, the outcome of this 324 study is useful to amend the ACL reconstruction treatment method. Due to the results of the 
325

326

327

328

329

330

331

332

333

334

335

336

337

338

339

340

341

optimum diameter of core bone, BASHTI technique can be an alternative ACL reconstruction method for patients.

\section{References}

1. Fleming BC, Hulstyn MJ, Oksendahl HL, Fadale PD. Ligament Injury, Reconstruction and Osteoarthritis Current opinion in orthopaedics 2005;16(5):354-62.

2. DiFelice GS, Villegas C, Taylor S. Anterior Cruciate Ligament Preservation: Early Results of a Novel Arthroscopic Technique for Suture Anchor Primary Anterior Cruciate Ligament Repair http://dx.doi.org/10.1016/j.arthro.2015.08.010

3. Mayr R, Heinz C, Martin H, Vinzenz E, Schmoelz W, Attal R. Preparation techniques for all - inside ACL cortical button grafts: a biomechanical study http://dx.doi.org/10.1007/s00167-015-3605-9

4. Shen X, Qu F, Li C, Qi W, Lu X, Li H, et al. Comparison between a novel human cortical bone screw and bioabsorbable interference screw for graft fixation of ACL reconstruction European Review for Medical and Pharmacological Sciences 2018;22:111-18.

5. Brown $\mathrm{CH}$, Hecker AT, Hipp JA, Myers ER, Hayes WC. The biomechanics of interference screw fixation of patellar tendon anterior cruciate ligament grafts https://journals.sagepub.com/doi/abs/10.1177/036354659302100622

6. Chizari M, Wang B, Snow M, Barrett M. Experimental and Numerical Analysis of Screw Fixation in Anterior Cruciate Ligament Reconstruction Experimental and Numerical Analysis of Screw Fixation in Anterior Cruciate Ligament Reconstruction In: AIP 
Conference Proceedings ; 2008. pp. 61-70.

347 7. Daneshvarhashjin N, Chizari M, Mortazavi J, Rouhi G. Can the body slope of interference screw affect initial stability of reconstructed anterior cruciate ligament?: An in-vitro investigation https://doi.org/10.1186/s12891-021-04446-8

350

351

8. Saithna A, Chizari M, Morris G, Anley C, Wang B, Snow M. An analysis of the biomechanics of interference screw fixation and sheathed devices for biceps tenodesis http://dx.doi.org/10.1016/j.clinbiomech.2015.04.006

9. Borjali A, Mohseni M, Chizari M. Biomechanical Modeling of a Bone Tunnel Enlargement Post ACL Reconstruction http://biorxiv.org/content/early/2020/09/04/2020.09.03.281915.abstract

10. Kulczycka P, Larbi A, Malghem J, Thienpont E, Berg B Vande, Lecouvet F. Imaging ACL reconstructions and their complications http://dx.doi.org/10.1016/j.diii.2014.04.007

11. Biazzo A, Manzotti A, Motavalli K, Confalonieri N. Femoral press-fit fixation versus interference screw fixation in anterior cruciate ligament reconstruction with bone-patellar tendon-bone autograft: 20-year follow-up https://doi.org/10.1016/j.jcot.2018.02.010

12. Barié A, Köpf M, Jaber A, Moradi B, Schmitt H, Huber J, et al. Long-term follow-up after anterior cruciate ligament reconstruction using a press-fit quadriceps tendon-patellar bone autograft https://bmcmusculoskeletdisord.biomedcentral.com/articles/10.1186/s12891$018-2271-8$

13. Bashti K, Tahmasebi MN, Kaseb H, Farahmand F, Akbar M, Mobini A. Biomechanical Comparison Between Bashti Bone Plug Technique and Biodegradable Screw for Fixation 
of Grafts in Ligament surgery Archives of Bone and Joint Surgery 2015;3(1):29-34.

14. Moeinnia H, Nourani A, Borjali A, Mohseni M, Ghias N, Korani H, et al. Effect of Geometry on the Fixation Strength of Anterior Cruciate Ligament Reconstruction Using BASHTI Technique https://www.thiemeconnect.com/products/ejournals/abstract/10.1055/s-0040-1716371

15. Borjali A, Farrahi G, Jafarzade H, Chizari M. Experimental study of a sheathed core bone plug in Bashti ACL reconstructive method In: The 27th Annual International Conference of Iranian Society of Mechanical Engineers-ISME2019; 2019

16. Nourani A, Mohseni M, Korani H, Ghias N, Chizari M. Reconstruction of a long head biceps using Bashti method; comparison between two different insertion techniques In: The 27th Annual International Conference of Iranian Society of Mechanical EngineersISME2019; 2019

17. Beynnon BD, Amis AA. In vitro testing protocols for the cruciate ligaments and ligament reconstructions http://link.springer.com/10.1007/s001670050226

18. Chizari M, Wang B, Barrett M, Snow M. BIOMECHANICAL TESTING PROCEDURES IN TENDON GRAFT RECONSTRUCTIVE ACL SURGERY https://www.worldscientific.com/doi/abs/10.4015/S1016237210002195

19. Snow M, Cheung W, Mahmud J, Evans S, Holt C, Wang B, et al. Mechanical assessment of two different methods of tripling hamstring tendons when using suspensory fixation http://link.springer.com/10.1007/s00167-011-1619-5

20. Kousa P, Järvinen TLN, Vihavainen M, Kannus P, Järvinen M. The Fixation Strength of 
Six Hamstring Tendon Graft Fixation Devices in Anterior Cruciate Ligament

391 21. Amis AA, Dawkins GP. Functional anatomy of the anterior cruciate ligament. Fibre bundle actions related to ligament replacements and injuries. The Journal of bone and joint surgery British volume 1991;73(2):260-67.

22. Lim B, Shin H, Lee Y. Biomechanical comparison of rotational activities between anterior sports traumatology, arthroscopy : official journal of the ESSKA 2014;23. 